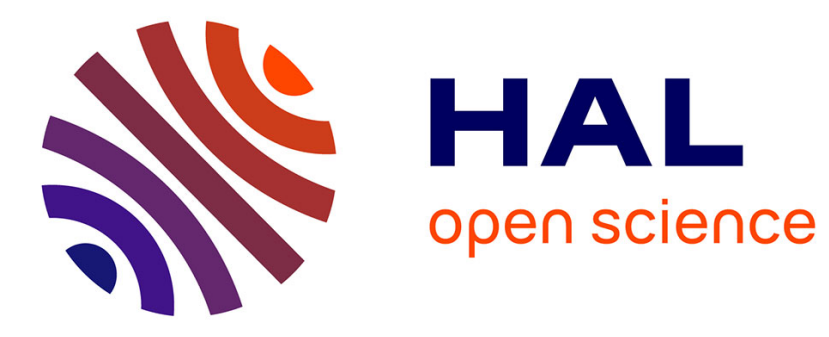

\title{
The generalized Doppler effect for surface waves \\ Guillaume Michel
}

\section{- To cite this version:}

Guillaume Michel. The generalized Doppler effect for surface waves. EPL - Europhysics Letters, 2016, 116 (4), pp.44002. 10.1209/0295-5075/116/44002 . hal-01494002

\section{HAL Id: hal-01494002 https: / hal.sorbonne-universite.fr/hal-01494002}

Submitted on 22 Mar 2017

HAL is a multi-disciplinary open access archive for the deposit and dissemination of scientific research documents, whether they are published or not. The documents may come from teaching and research institutions in France or abroad, or from public or private research centers.
L'archive ouverte pluridisciplinaire HAL, est destinée au dépôt et à la diffusion de documents scientifiques de niveau recherche, publiés ou non, émanant des établissements d'enseignement et de recherche français ou étrangers, des laboratoires publics ou privés. 


\title{
The generalized Doppler effect for surface waves
}

\author{
G. MiCHEL \\ 1 Laboratoire de Physique Statistique, Ecole Normale Supérieure, CNRS, Université P. et M. Curie, Université Paris \\ Diderot, Paris, France
}

\begin{abstract}
PACS 42.25.Gy - Edge and boundary effects; reflection and refraction
PACS 47.35. Bb - Gravity waves (Hydrodynamic waves)

PACS 43.20.+g - General linear acoustics
\end{abstract}

\begin{abstract}
We investigate energy exchanges through scales occurring when a surface wave reflects on a harmonically oscillating wall. We first experimentally evidence the creation of Dopplershifted waves and measure their height as a function of the oscillation amplitude. Then, we theoretically compute the amplitudes of these new waves in the gravity regime. Both results show that even without bulk non-linearities, oscillating paddles in a fluid container lead to a complex wave energy spectrum competing with the one predicted by wave turbulence. To exemplify this point, we characterize a simple one-dimensional model consisting of a linear wave equation in an oscillating cavity with distinct injection and dissipation mechanisms. It displays features usually associated with non-linearities, as self-similarity in a spectral domain (the so-called inertial range), appearance of energy at larger and/or lower scales than the forcing one and creation of shock waves.
\end{abstract}

Introduction. - The reflection of a wave on a moving obstacle can be investigated from the usual wave equation and leads for a constant velocity to the well-known Doppler effect, a frequency shift between the incident and reflected waves. This can be generalized to any motion of the boundary, as, for instance, a vibration around a fixed position. In the case of a harmonic oscillation at frequency $f_{\mathrm{w}}$, Censor showed that the spectrum of the reflected wave is of the form $f_{0}+n f_{\mathrm{w}}, f_{0}$ being the frequency of the incident wave and $n$ any integer [1]. Since this result is obtained from the wave equation, it holds both in electrodynamics and in mechanics. It has been discussed in detail in acoustics through a controversy about the comparative effect of the medium non-linearity (see [2] and references therein) and later confirmed by experiments [3]. Surface waves, on the other hand, cannot be described by a wave equation when interactions with boundaries are considered. This results from the fact that the normal velocity of the fluid has to be prescribed on the entire solid surface: thus, the motion of a vertical wall creates surface waves, whereas the one of a perfect mirror does not give rise to any electric field (in the classical theory and if no field is initially present).

The reflection of a surface wave on an oscillating wall is therefore a quite different problem from the one of electromagnetic/mechanical waves and it has so far never been considered. We start this study by experimentally evidencing that the spectrum of a monochromatic surface wave is broadened by such an interaction. The first corrections are found to grow linearly with the oscillation amplitude of the wall. We then theoretically compute these corrections for gravity surface waves. This shed light on three characteristics of the generalized Doppler effect. (i) Surface waves undergo sizable energy exchanges between scales at the reflection on an oscillating boundary. (ii) This process is linear with respect to the surface elevation, i.e., it does not involve the non-linear term of the Navier-Stokes equation. (iii) In some limits, the firstorder correction is similar to the one obtained with the usual wave equation.

Given these results, consider a typical setup for the study of surface wave turbulence using wave-makers, that are oscillating paddles (see, e.g., [4]). In the usual reading of such experiments, wave-makers inject energy in a given bandwidth and bulk non-linearities lead to energy exchanges between waves. The phenomenology, close to the one of hydrodynamic turbulence and involving direct and/or inverse cascades, is called wave turbulence [5]. According to the discussion above, it seems natural to wonder if such states could be notably altered by the continuous bouncing of surface waves on the wave-makers. This question turns out to be difficult to address since both bulk 
non-linearities and oscillating boundaries would have to be considered simultaneously. We rather consider the following problem: what is the steady-state energy spectrum of linear waves trapped in an oscillating cavity?

In the absence of dissipation and if the motion of the wall is sinusoidal at a frequency close to twice an eigenfrequency of the cavity, it has been shown that the energy eventually diverges as a consequence of a parametric resonance [6-8]. This has received considerable attention in quantum electrodynamics as it occurs even if the initial state is the vacuum, hence creating photons from zero point fluctuations (see the review [9] and references therein). From a practical point of view, the motion of the mirror becomes more and more difficult to sustain, a phenomenon called dynamical Casimir effect.

To be in line with the phenomenology of wave turbulence, we differ from these studies and consider distinct injection and dissipation mechanisms. We report that: (i) Interactions with the moving boundary may inject energy at larger and/or lower scales than the forcing one. (ii) Self-similar energy spectra can be observed over a large frequency range. (iii) Shock waves can be formed. We emphasize that all these features, usually associated with non-linearities, result in this system from linear processes.

Experimental measurement. - We first experimentally characterize the spectrum of a harmonic surface wave that reflects on an oscillating wall, using a recent measurement technique able to disentangle between counterpropagating waves of identical frequencies [10]. The setup is sketched in fig. 1 and consists of a basin of dimension $648 \times 846 \times 160 \mathrm{~mm}$ filled with water up to $130 \mathrm{~mm}$, one wave-maker being fixed at the left extremity (denoted as WM1) and another one at the right extremity (WM2). Each of them (oscillating paddle of size $120 \times 80 \times 4 \mathrm{~mm}$ plunged $60 \mathrm{~mm}$ below the free surface) is driven harmonically by a Brüel \& Kjær 4810 shaker and its motion is tracked with a Brüel \& Kjær 4393 accelerometer. WM1 oscillates at $f_{1}=15 \mathrm{~Hz}$ and WM2 at $f_{2}=3 \mathrm{~Hz}$.

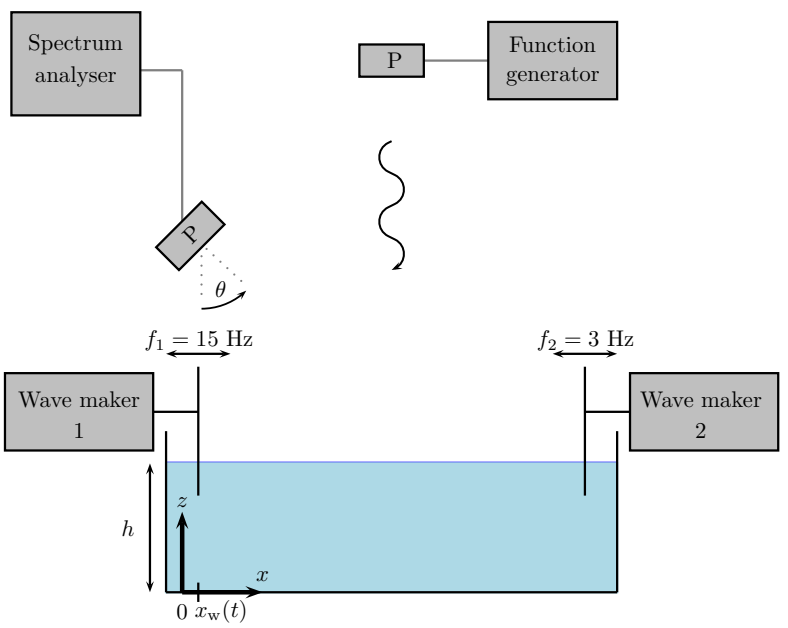

Fig. 1: Experimental setup for the measurement of surface waves. $P$ stands for piezoelectric transducer.
Two piezoelectric transducers resonant at $f_{0}=41500 \mathrm{~Hz}$ are used: one emits a harmonic wave of normal incidence and the other receives at $\theta=45.2^{\circ}$ the acoustic signal, then processed by a HP 35670A spectrum analyzer. Surface waves can be fully characterized by this scattered signal, the entire setup being equivalent to an acousto-optic modulator [10]. For the upcoming discussion to be clear, we sum up the main features of this technique:

1. If the scattered signal includes a component of frequency $f_{0}+f_{\mathrm{w}}$ and amplitude $A_{\mathrm{w}}$, then the insonified area is crossed by a monochromatic wave, heading toward the receiver (i.e., in the direction of WM1), of frequency $f_{\mathrm{w}}$ and height $\eta_{\mathrm{w}}=C\left(\left|f_{\mathrm{w}}\right|\right) A_{\mathrm{w}}$. $C$ is a function that can be experimentally measured.

2. If the scattered signal includes a component of frequency $f_{0}-f_{\mathrm{w}}$ and amplitude $A_{\mathrm{w}}$, then the insonified area is crossed by a monochromatic wave, heading away from the receiver (i.e., in the direction of WM2), of frequency $f_{\mathrm{w}}$ and height $\eta_{\mathrm{w}}=C\left(\left|f_{\mathrm{w}}\right|\right) A_{\mathrm{w}}$.

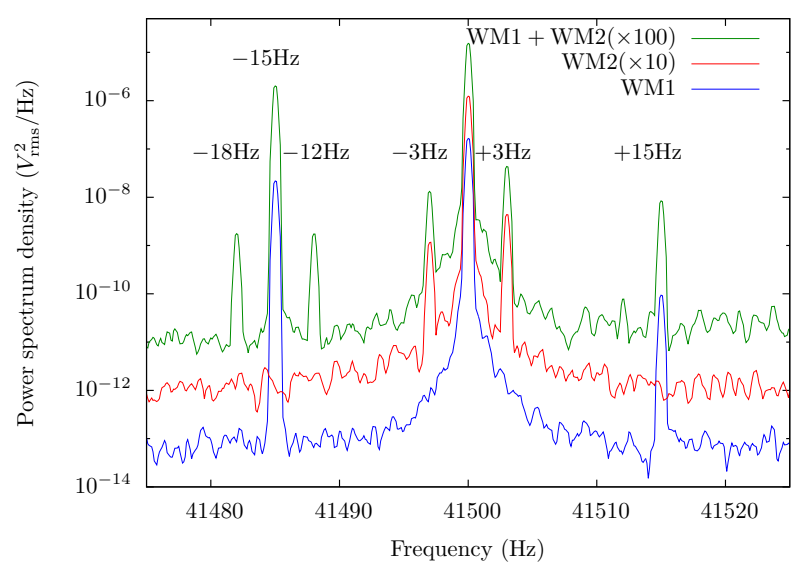

Fig. 2: Spectra obtained for one or two oscillating wave-makers. When active, the oscillation amplitude of WM1 is $56 \mu \mathrm{m}$.

We then comment on the spectra shown in fig. 2 :

- If only WM1 is active (blue lower curve of fig. 2), the measurement area is dominated by progressive waves of frequency $f_{1}=15 \mathrm{~Hz}$ heading away from WM1. A counterpropagating wave is distinguishable and comes from the reflection on WM2 (at rest).

- If only WM2 oscillates (middle red curve of fig. 2), the situation is reversed and waves of frequency $f_{2}=$ $3 \mathrm{~Hz}$ are observed. Note that unlike the previous ones, these waves are almost of similar amplitude, a consequence of the damping frequency dependence.

- If both WM2 and WM1 are in motion (upper green curve of fig. 2), the scattered acoustic spectrum does not reduce to the superposition of the last two spectra: two components of frequency $f_{1} \pm f_{2}$, leaving WM1 are also observed. Waves at $f_{2} \pm f_{1}$ that would also be expected are below the noise level. 
This demonstrates that the reflection of a progressive wave of frequency $f_{2}=3 \mathrm{~Hz}$ on a vertical wall oscillating at $f_{1}=$ $15 \mathrm{~Hz}$ creates two new components of frequency $f_{2} \pm f_{1}$. Moreover, their amplitudes are directly proportional to the wall oscillation one, see fig. 3. This is reminiscent of the reflection of an electromagnetic/acoustic wave of wave number $k$ on a scatterer of oscillation amplitude $\delta$ [11]: if $\delta k \ll 1$, the Doppler effect reduces to these two sidebands, whose amplitude linearly grows with $\delta k$. In the present experiment, $\delta k<6.10^{-3}$ with $k=36 \mathrm{~m}^{-1}$ based on $f_{2}$. Finally, note that the amplitudes of these Doppler-shifted waves have been measured up to the function $C\left(\left|f_{\mathrm{w}}\right|\right)$, and that they have been reduced by another damping factor depending on their frequencies on their way from WM1 to the insonified area: the overlapping of the curves in fig. 3 is therefore fortuitous.

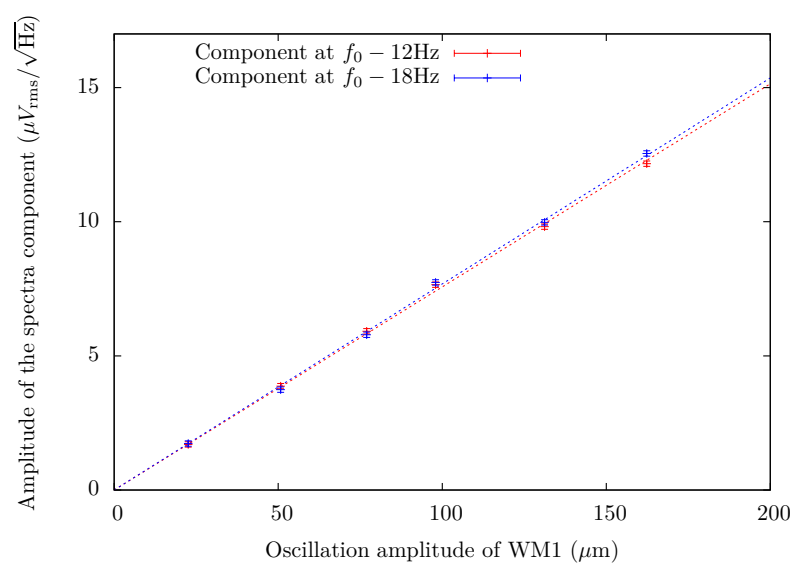

Fig. 3: Growth of the sidebands with the oscillation amplitude.

Generalized Doppler effect for gravity waves. To provide a theoretical support to these observations, we compute in this section the amplitudes of these sidebands. We restrict to gravity waves and thus disregard the complex meniscus dynamics. This only approximately models the previous experiment that involves gravity capillary waves. Moreover, we consider the limit of inviscid fluid, small wave steepness and small oscillation amplitude so that both viscosity and bulk non-linearities can be dropped from the Navier-Stokes equation.

Let $\vec{v}=-\vec{\nabla} \phi$ be the total velocity field, $x_{\mathrm{w}}(t)=$ $\delta \sin \left(\omega_{\mathrm{w}} t\right)$ the wall position and $h$ the height of the fluid. Given that non-linearities are neglected, the potential $\phi$ is

$$
\phi(\vec{r}, t)=\phi_{\mathrm{w}}(x, z, t)+\phi_{\mathrm{inc}}(x, z, t)+\phi_{\mathrm{ref}}(x, z, t),
$$

where $\phi_{\mathrm{w}}$ describes the flow induced by the wall motion in the absence of any external wave, and $\phi_{\text {inc }}$ and $\phi_{\text {ref }}$ stand for these additional incident and reflected waves. $x$ and $z$ are the horizontal and vertical directions, such that at rest the fluid lies within $(x \geqslant 0, h \geqslant z \geqslant 0)$. Within our assumptions, $\phi$ verifies

$$
\begin{aligned}
& \partial_{x x} \phi(x, z, t)+\partial_{z z} \phi(x, z, t)=0 \\
& \partial_{z} \phi(x, z=0, t)=0 \\
& \partial_{t t} \phi(x, z=h, t)=-g \partial_{z} \phi(x, z=h, t) \\
& \partial_{x} \phi\left(x=x_{\mathrm{w}}(t), z, t\right)=-\dot{x}_{\mathrm{w}}(t)
\end{aligned}
$$

We consider an incident progressive plane wave of angular frequency $\omega_{0}$ and of velocity potential

$$
\begin{aligned}
& \phi_{\text {inc }}(x, z, t)=\Psi_{\text {inc }} \cosh \left(k_{0} z\right) e^{i\left(\omega_{0} t+k_{0} x\right)} \\
& \omega_{0}^{2}=g k_{0} \tanh \left(k_{0} h\right)
\end{aligned}
$$

The computation of $\phi_{\mathrm{w}}$ has been first carried out by Havelock in 1929 for gravity waves [12], and it provides an explicit expression for the amplitudes of free waves emitted by a wave-maker. Additional effects such as capillarity have been later considered, see [13] and references therein. This potential is such that

$$
\partial_{x} \phi_{\mathrm{w}}\left(x=x_{\mathrm{w}}(t), z, t\right)=-\dot{x}_{\mathrm{w}}(t),
$$

and the boundary condition fulfilled by $\phi_{\text {ref }}$ thus reads

$$
\begin{aligned}
& \partial_{x x} \phi_{\mathrm{ref}}(x, z, t)+\partial_{z z} \phi_{\mathrm{ref}}(x, z, t)=0 \\
& \partial_{z} \phi_{\mathrm{ref}}(x, z=0, t)=0 \\
& \partial_{t t} \phi_{\mathrm{ref}}(x, z=h, t)=-g \partial_{z} \phi_{\mathrm{ref}}(x, z=h, t) \\
& \partial_{x} \phi_{\mathrm{ref}}\left(x=x_{\mathrm{w}}(t), z, t\right)=-\partial_{x} \phi_{\mathrm{inc}}\left(x=x_{\mathrm{w}}(t), z, t\right)
\end{aligned}
$$

This last equation can be decomposed into a basis of harmonic functions using (6) and a first-order expansion in the small parameter $\left(k_{0} \delta\right)$ :

$$
\begin{aligned}
& \partial_{x} \phi_{\mathrm{ref}}\left(x=x_{\mathrm{w}}(t), z, t\right)= \\
& -i k_{0} \Psi_{\mathrm{inc}} \cosh \left(k_{0} z\right)\left(e^{i \omega_{0} t}+\frac{k_{0} \delta}{2} e^{i \omega_{1} t}-\frac{k_{0} \delta}{2} e^{i \omega_{-1} t}\right)
\end{aligned}
$$

with $\omega_{n}=\omega_{0}+n \omega_{\mathrm{w}}$. The method for solving this problem turns out to be very similar to the one used by Havelock to compute $\phi_{\mathrm{w}}$. The velocity potential we are looking for, $\phi_{\text {ref }}$, can not simply be expressed as a sum of free waves: even though (9 - 11) would be verified, (13) would not. In addition, evanescent waves have to be considered and the proper decomposition is

$$
\begin{aligned}
& \phi_{\mathrm{ref}}(x, z, t)=\sum_{n} \varphi_{n}(x, z, t)+\psi_{n}(x, z, t) \\
& \varphi_{n}(x, z, t)=\varphi_{n} \cosh \left(k_{n} z\right) e^{i\left(\omega_{n} t-k_{n} x\right)} \\
& \psi_{n}(x, z, t)=\sum_{m} \psi_{n, m} \cos \left(\tilde{k}_{n, m} z\right) e^{i \omega_{n} t-\tilde{k}_{n, m} x} \\
& \omega_{n}^{2}=g k_{n} \tanh \left(k_{n} h\right)=-g \tilde{k}_{n, m} \tan \left(\tilde{k}_{n, m} h\right)
\end{aligned}
$$

With these expressions, $(9-11)$ are directly fulfilled and (13) is the last remaining constraint. Note that for $\delta=0$, $\varphi_{0}=\Psi_{\text {inc }}$ while $\varphi_{n \neq 0}$ and $\psi_{n, m}$ vanish and are therefore of first order (or more) in $\left(k_{0} \delta\right)$. $\partial_{x} \phi_{\text {ref }}\left(x=x_{\mathrm{w}}(t), z, t\right)$ is then expressed from (14-16), expanded in the first order of 
$\left(k_{0} \delta\right)$ and decomposed over the harmonic functions $e^{i \omega_{n} t}$ of (13). On $e^{i \omega_{1} t}$, we get

$$
\begin{aligned}
-i k_{0} \Psi_{\text {inc }} & \cosh \left(k_{0} z\right)\left(\frac{k_{0} \delta}{2}\right)=i k_{0} \varphi_{0} \cosh \left(k_{0} z\right)\left(\frac{k_{0} \delta}{2}\right) \\
& -i k_{1} \varphi_{1} \cosh \left(k_{1} z\right) \\
& +i \sum_{n, m} i^{n} \tilde{k}_{n, m} \psi_{n, m} \cos \left(\tilde{k}_{n, m} z\right) I_{1-n}\left(-\tilde{k}_{n, m} \delta\right)
\end{aligned}
$$

where $I_{n}$ are modified Bessel functions of the first kind. The integration $\int_{0}^{h}(18) \times \cosh \left(k_{1} z\right) \mathrm{d} z$ removes the contributions of evanescent waves at this order and leads to

$$
\begin{gathered}
k_{0}^{2} \delta \Psi_{\text {inc }} \frac{\cosh \left(k_{0} h\right) \cosh \left(k_{1} h\right)}{g\left(k_{1}^{2}-k_{0}^{2}\right)}\left(\omega_{1}^{2}-\omega_{0}^{2}\right)= \\
k_{1} \varphi_{1}\left(\frac{h}{2}+\frac{\sinh \left(2 k_{1} h\right)}{4 k_{1}}\right) .
\end{gathered}
$$

A similar expression is obtained for $\varphi_{-1}$. We sum up these results using the wave height $\eta$ (e.g., $\eta_{\text {inc }}=$ $\left.-k_{0} \Psi_{\text {inc }} \sinh \left(k_{0} h\right) / \omega_{0}\right)$ instead of the velocity potential $\left(e . g ., \Psi_{\text {inc }}\right)$ : a surface gravity wave $\eta_{\text {inc }} \sin \left(\omega_{0} t+k_{0} x\right)$ reflecting on a vertical wall at $x_{\mathrm{w}}(t)=\delta \sin \left(\omega_{\mathrm{w}} t\right)$ gives rise at the first order in $k_{0} \delta$ to a reflected free wave of the form

$\eta_{\text {inc }} \sin \left(\omega_{0} t-k_{0} x\right)+\eta_{1} \sin \left(\omega_{1} t-k_{1} x\right)+\eta_{-1} \sin \left(\omega_{-1} t-k_{-1} x\right)$

where $\omega_{ \pm 1}=\omega_{0} \pm \omega_{\mathrm{w}}$ and $k_{ \pm 1}$ are related to $\omega_{ \pm 1}$ via the dispersion relation. $\eta_{ \pm 1}$ are given by

$$
\begin{aligned}
\frac{\eta_{1}}{\eta_{\mathrm{inc}}} & =\left(k_{0} \delta\right) \frac{k_{0}\left(\omega_{1}^{2}-\omega_{0}^{2}\right)}{\omega_{0} \omega_{1} h\left(k_{1}^{2}-k_{0}^{2}\right)}\left(\frac{\sinh \left(2 k_{1} h\right)}{1+\frac{\sinh \left(2 k_{1} h\right)}{2 k_{1} h}}\right) \\
\frac{\eta_{-1}}{\eta_{\text {inc }}} & =-\left(k_{0} \delta\right) \frac{k_{0}\left(\omega_{0}^{2}-\omega_{-1}^{2}\right)}{\omega_{0} \omega_{-1} h\left(k_{0}^{2}-k_{-1}^{2}\right)}\left(\frac{\sinh \left(2 k_{-1} h\right)}{1+\frac{\sinh \left(2 k_{-1} h\right)}{2 k_{-1} h}}\right)
\end{aligned}
$$

The linear growth of the sidebands $\eta_{ \pm 1} \propto \delta$ is consistent with the experimental observations of fig. 3. The shallow water and deep water limits of (21) are

$$
\begin{aligned}
& \frac{\eta_{1}}{\eta_{\text {inc }}} \underset{h \rightarrow 0}{=} k_{0} \delta \\
& \frac{\eta_{1}}{\eta_{\text {inc }}} \underset{h \rightarrow \infty}{=} 2 k_{0} \delta \frac{\omega_{0} \omega_{1}}{\omega_{0}^{2}+\omega_{1}^{2}} \underset{\omega_{\mathrm{w}} \ll \omega_{0}}{=} k_{0} \delta
\end{aligned}
$$

In acoustics and electromagnetism, $\eta$ obeys the wave equation and vanishes at the moving boundary. In the limit of small oscillations, Censor computed $\eta_{ \pm 1} / \eta_{\text {inc }}= \pm k_{0} \delta[11]$, i.e., exactly what is found both for deep water with small oscillation frequency and shallow water. Therefore, to describe the Doppler effect in these limits, surface waves can be modelled by the wave equation with a pinned contact line. This is natural for shallow water, since $v_{x}$ becomes in this limit independent of $z$ and directly proportional to the wave height $\eta$. However, we can think of no way to anticipate this result for deep water. In order to extend this calculation to gravito-capillary waves, one should take into account the meniscus, whose specific dynamics when undergoing an oscillating motion is an active research area, see e.g., [14]. Phenomenological boundary conditions may be used, as has been done for the wave-maker problem [13] or for the reflection of a gravity capillary surface wave on a steady wall [15]. We also point out that the additional work required to sustain the wall oscillation, crucial to discuss energy exchanges, could be derived by comparing the incident and reflected waves energies. The second-order correction at the incident wave frequency $\omega_{0}$ would then have to be worked out.

Wave energy transfer in an oscillating cavity. So far, we have focused on the simplest configuration of a monochromatic wave reflecting on a harmonically oscillating wall. However, most experiments dealing with surface waves are conducted in closed basin without any specific care to damp reflected waves, as a beach would do. This is especially relevant in wave turbulence, where dissipation is sought as small as possible, hence the use of fluids with low kinematic viscosity or pinned contact line on the steady boundaries. Moreover, a typical way of creating surface waves is to use a wave-maker, which is an oscillating wall (other geometries exist, as flaps hinged below the free surface). In most experiments of wave turbulence, its motion is not harmonic but consists of a filtered noise. Therefore, waves emitted by the wave-maker reflect a large number of time on it until they are eventually damped. The steady state associated with these cumulative Doppler effects has so far never been investigated in this context and we propose a simple model expected to capture its main features.

As a first approach, we consider a one-dimensional dissipative and linear wave equation for a perturbation $\eta(x, t)$,

$$
\frac{\partial^{2} \eta}{\partial t^{2}}+\sigma \frac{\partial \eta}{\partial t}=c^{2} \frac{\partial \eta^{2}}{\partial x^{2}}
$$

where $c$ is the wave velocity and $\sigma$ the dissipation coefficient. The use of a wave equation is reasonable since firstorder corrections have been found similar in some limits. However, even if non-linear interactions between waves are more restricted in hydrodynamics than in acoustics (it respectively involves three or four waves processes even more in one dimension vs. two-waves processes), it should be kept in mind that sharp structures that shall arise would clearly lead to non-linear dynamics. Equation (25) also neglects dispersion of water waves, since $c$ does not depend on the frequency. The boundary conditions are

$$
\begin{aligned}
& \eta(0, t)=\eta_{\mathrm{f}} \sin \left(n_{\mathrm{f}} \omega t\right), \quad\left(\omega=\frac{\pi c}{L}, n_{\mathrm{f}} \in \mathbb{N}\right) \\
& \eta(L+\xi(t))=0,
\end{aligned}
$$

eq. (26) corresponds to an energy injection by an external operator at a resonance frequency and eq. (27) is the reflection on the moving boundary, the cavity being of mean length $L$ modulated by $\xi(t)$. The energy balance can be derived directly from these equations. Defining the energy by $E(t)=\frac{1}{2} \int_{0}^{L+\xi(t)} \mathrm{d} x\left(\left(\partial_{t} \eta\right)^{2}+c^{2}\left(\partial_{x} \eta\right)^{2}\right)$, we get 


$$
\begin{aligned}
\frac{\mathrm{dE}}{\mathrm{d} t}= & -\sigma \int_{0}^{L+\xi(t)} \mathrm{d} x\left(\frac{\partial \eta}{\partial t}\right)^{2} \\
& -c^{2}\left(\frac{\partial \eta}{\partial t}\right)(0, t)\left(\frac{\partial \eta}{\partial x}\right)(0, t) \\
& -\xi^{\prime}(t) \frac{\left(c^{2}-\xi^{\prime}(t)^{2}\right)}{2}\left(\frac{\partial \eta}{\partial x}\right)^{2}(L+\xi(t), t),
\end{aligned}
$$

where the terms are, respectively, dissipation, work done by the operator and interaction with the moving wall via radiative pressure. In a steady-state, the mean value of (28) vanishes and dissipation is balanced by injection of energy by the operator and by the motion of the wall. Thereafter periodic and stochastic oscillations of the cavity are considered successively.

The periodic motion. - We first consider a lowfrequency periodic motion of the wall,

$$
\xi(t)=\xi_{\mathrm{w}} \sin (\omega t)
$$

with $\omega=\frac{\pi c}{L}$ the first eigenfrequency of the cavity. The solution will be found at first order in the two dimensionless parameters $\mathcal{D}=\sigma L / c$ and $\epsilon=\pi \xi_{\mathrm{w}} / L$. We define the ratio of these numbers as $\alpha=\mathcal{D} / \epsilon$, roughly the ratio of dissipation to the oscillation amplitude. Note that the Mach number Ma, representing the ratio of the wall velocity to the speed of the waves, remains smaller than one:

$$
\mathrm{Ma}=\frac{\xi_{\mathrm{w}} \omega}{c}=\epsilon \ll 1 .
$$

We look for a periodic steady state of period $2 L / c$. If $\epsilon=0$, the wall is at rest and the solution is $\eta(x, t)=$ $\eta_{\mathrm{f}} \eta_{+}(x, t)$ with

$$
\begin{aligned}
\eta_{+}(x, t)=\frac{2}{\mathcal{D}}( & -\sin \left(\frac{n_{\mathrm{f}} \omega x}{c}\right) \cos \left(n_{\mathrm{f}} \omega t\right) \\
& \left.+\frac{\mathcal{D}}{2}\left(1-\frac{x}{L}\right) \cos \left(\frac{n_{\mathrm{f}} \omega x}{c}\right) \sin \left(n_{\mathrm{f}} \omega t\right)\right) .
\end{aligned}
$$

If $\epsilon>0$, the steady state is of the form $\eta(x, t)=$ $\eta_{\mathrm{f}}\left(\eta_{+}(x, t)+\sum_{n} A_{n} \eta_{n}(x, t)\right)$, with $\eta_{n}(x, t)$ other eigenmodes of (25),

$$
\begin{aligned}
\eta_{n}(x, t)=\frac{2}{\mathcal{D}}( & \sin \left(\frac{n \omega x}{c}\right) \cos (n \omega t) \\
& \left.+\frac{\mathcal{D} x}{2 L} \cos \left(\frac{n \omega x}{c}\right) \sin (n \omega t)\right) .
\end{aligned}
$$

This solution verifies (25) and (26). Equation (27) fixes the constants $\left\{A_{n}\right\}$ via a recurrence relation. It has a simple form if only the first modes are considered $(n \ll$ $\left.\epsilon^{-1}\right)$, that is with $\delta_{i}^{j}$ the Kronecker delta

$-A_{n-1}(n-1)+\alpha A_{n}+A_{n+1}(n+1)=n_{\mathrm{f}}\left(\delta_{n}^{n_{\mathrm{f}}-1}-\delta_{n}^{n_{\mathrm{f}}+1}\right)$.

In this range of parameters, we get local interactions, i.e., mode coupling restricted to neighbors in the spectral domain. This is directly related to the work of Censor mentioned in the introduction [1] and to our previous study: if a wave of frequency $f_{0}$ and wave number $k_{0}$ reflects on a moving boundary oscillating at a frequency $f_{\mathrm{w}}$ and amplitude $\xi_{\mathrm{w}} \ll k_{0}^{-1}$, sidebands of frequency $f_{0} \pm f_{\mathrm{w}}$ appear. Conversely, if $\xi_{\mathrm{w}} k_{0} \gtrsim 1$, harmonics at $f_{0} \pm n f_{\mathrm{w}}$ $(n \geqslant 2)$ are of similar amplitudes as the ones at $f_{0} \pm f_{\mathrm{w}}$. Modes at $n \gtrsim \epsilon^{-1}$ therefore lead to non-local interactions and are neglected in this study.

Equation (33) can be solved for any value of $n_{\mathrm{f}}$, and we will detail low- and high-frequency forcing. It can be seen as the equilibrium state of a shell model with local linear coupling between modes (shell models are phenomenological equations used to describe systems with non-linear coupling between modes, e.g., turbulent states [16]).

Low-frequency forcing: $n_{\mathrm{f}}=1$. We derive the following expressions for the constants $\left\{A_{n}\right\}$ :

$$
\begin{aligned}
A_{n} \sim \frac{(n-1) !}{\alpha^{n-1}}, & (2 \leqslant n \ll \alpha) \\
A_{n} \sim n^{-1-\frac{\alpha}{2}} . & \left(\alpha \ll n \ll \epsilon^{-1}\right)
\end{aligned}
$$

Given that the energy of the $n$-th mode is $E_{n} \sim\left(n A_{n}\right)^{2}$, it displays a self-similar range for $\alpha \ll n \ll \epsilon^{-1}$, where $E_{n} \sim$ $n^{-\alpha}$. In the non-dissipative limit, the energy spectrum becomes independent of $n$. Figure 4 shows examples of such spectra obtained by solving (33) numerically for $\alpha=$ 20 and $\alpha=40$. Approximate expressions (34) and (35) are superimposed. Since eq. (33) is local in frequency, the dissipation can be made frequency dependant $(\alpha \rightarrow \alpha(n))$ : a dissipation-free range is also displayed, obtained with $\alpha(n)=0$ if $n \in[10,100]$ and 10 otherwise.

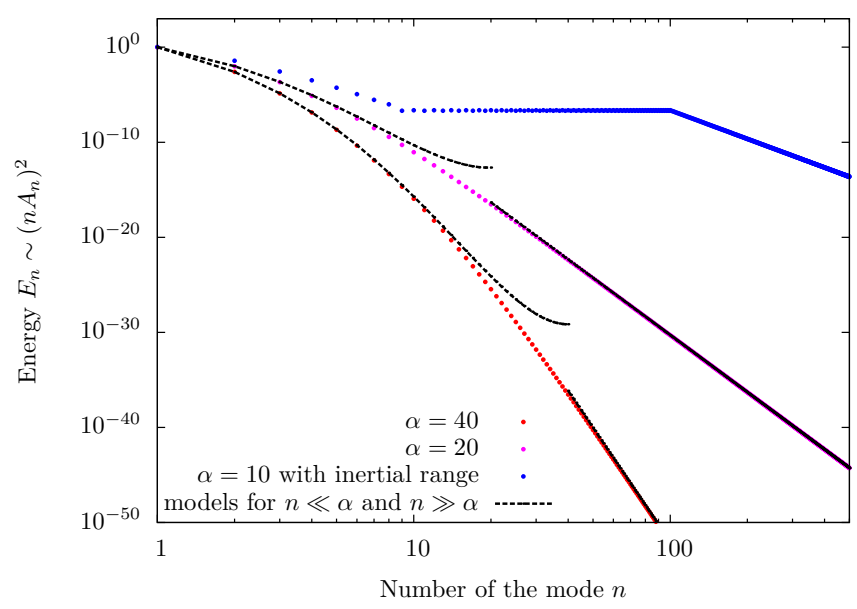

Fig. 4: Spectra for the low-frequency forcing. Several values of $\alpha$ are reported, with the approximate expressions (34-35). For $\alpha=10$, dissipation is taken equal to zero in the range $n \in[10,100]$.

As the dissipation vanishes or as the amplitude of the motion increases, i.e., in the limit $\alpha \rightarrow 0$, a shock wave is formed. In this limit, the additional wave field reduces to (according to (35)),

$$
\eta_{\mathrm{f}} \sum_{n} A_{n} \eta_{n}(x, t) \underset{\alpha \rightarrow 0}{\sim} \frac{2 \eta_{\mathrm{f}}}{\mathcal{D}} \sum_{n} \frac{\sin \left(\frac{n \omega x}{c}\right) \cos (n \omega t)}{n} .
$$


The right-hand side (RHS) of (36) describes a sawtooth wave bouncing between the walls of the cavity at a celerity c. It is shown in fig. 5 for two values of $t$.

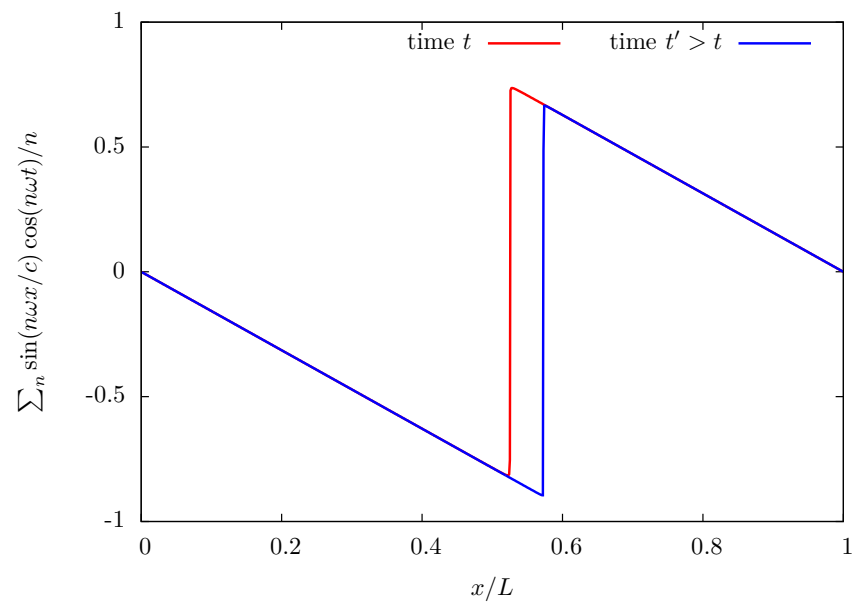

Fig. 5: Discontinuous wave field induced by the periodic motion of the boundary in the limit $\alpha \rightarrow 0$.

High-frequency forcing: $\alpha \ll n_{\mathrm{f}} \ll \epsilon^{-1}$. The coefficients $\left\{A_{n}\right\}$ have these expressions,

$$
\begin{array}{llrl}
A_{n} & \sim \frac{(-1)^{n-1} \alpha^{n-1}}{n !}, & & (2 \leqslant n \ll \alpha) \\
A_{n} \sim(-1)^{n-1} n^{-1+\frac{\alpha}{2}}, & & \left(\alpha \ll n \leqslant n_{\mathrm{f}}-1\right) \\
A_{n} \sim n^{-1-\frac{\alpha}{2}} . & & \left(n_{\mathrm{f}}+1 \leqslant n \ll \epsilon\right)
\end{array}
$$

The scaling law for the energy at frequencies larger than the forcing one is the same as before. However, we note that energy can be injected to frequencies lower than the forcing one with the Doppler effect, where it gets eventually either dissipated or stored depending on the local value of $\alpha$.

The situation in which dissipation vanishes at scales larger than the forcing one is of particular interest. In this case, energy is neither injected nor dissipated at these scales and the energy spectrum is found constant: this may correspond to a thermal equilibrium. It has for instance been numerically observed in hydrodynamic turbulence [17]. However, the present state turns out to be very different from an equilibrium one because of phase coupling between modes: strong gradients are formed that would result in a shock wave as the number of modes involved increases.

The stochastic motion. - In the previous section, we showed how the ground state (31) was affected by a periodic modulation of the cavity length. We now turn to a noisy motion of the boundary $\xi(t)$, whose one-sided power spectral density is denoted by $S_{\mathrm{w}}(\tilde{w})$. We still consider the limit of low dissipation $(\mathcal{D} \ll 1)$ and small motion of the wall $\left(\left\langle\xi(t)^{2}\right\rangle \ll(n \omega / c)^{-2}\right.$ for the modes considered). The wave field can be approximated as a sum of the motionless solution and an induced perturbation:

$$
\eta(x, t)=\eta_{\mathrm{f}}\left(\eta_{+}(x, t)+\sum_{n} \bar{\eta}_{n}(t) \sin \left(\frac{n \pi x}{L}\right)\right)
$$

Integrating the wave equation (25) times $\sin \left(\frac{n \pi x}{L}\right)$ on the entire domain leads in the mentioned limits to an equation for $\bar{\eta}_{n}(t)\left(n \neq n_{\mathrm{f}}\right)$ :

$\frac{\mathrm{d}^{2} \bar{\eta}_{n}}{\mathrm{~d} t^{2}}+\sigma \frac{\mathrm{d} \bar{\eta}_{n}}{\mathrm{~d} t}+(n \omega)^{2} \bar{\eta}_{n}=\frac{2 n \omega c}{L \eta_{\mathrm{f}}}(\eta(0, t)-\eta(L, t)) \cos (n \pi)$

Given that the quality factor of this damped harmonic oscillator $n \pi / \mathcal{D}$ is very large, out-of-resonance forcing is inefficient. The spectral component at angular frequency $n \omega$ of the left-hand side (LHS) of (41), governing the dynamics of $\bar{\eta}_{n}$, is found by expanding the boundary condition (27):

$$
\begin{aligned}
& \eta(L, t) \simeq-\xi(t) \frac{\partial \eta}{\partial x}(L, t) \\
& \simeq-\eta_{\mathrm{f}} \xi(t) \frac{\partial}{\partial x}\left(\eta_{+}(x, t)+\sum_{m} \bar{\eta}_{m}(t) \sin \left(\frac{m \pi x}{L}\right)\right)(L, t)
\end{aligned}
$$

Equation (43) provides two kinds of resonant terms. The first one results from interactions between the motion of the wall $\xi(t)$ and the induced perturbations $\left\{\bar{\eta}_{m}(t)\right\}$, hence generating a coupling between all oscillators. The second one is direct interaction with the forcing mode $\eta_{+}(x, t)$. Depending on the spectrum of $\xi(t)$, especially on the amplitudes of high frequencies, one of this forcing term will dominate the other. We restrict this study to coupling with $\eta_{+}(x, t)$, i.e., to large-band stochastic motion $\xi(t)$, as would be a thermal motion (relevant for applications in electromagnetism discussed in the conclusion) or a poorly filtered bandwidth noise. The dynamics of $\bar{\eta}_{n}$ is then ruled by

$$
\frac{\mathrm{d}^{2} \bar{\eta}_{n}}{\mathrm{~d} t^{2}}+\sigma \frac{\mathrm{d} \bar{\eta}_{n}}{\mathrm{~d} t}+(n \omega)^{2} \bar{\eta}_{n}=\frac{4 s(n \omega)\left(n_{\mathrm{f}} \omega\right) \xi(t) \cos \left(n_{\mathrm{f}} \omega t\right)}{L \mathcal{D}}
$$

with $s=-\cos (n \pi) \cos \left(n_{\mathrm{f}} \pi\right)= \pm 1$. As mentioned above, the high quality factor of the oscillator makes the dynamics insensitive to the components of the noise that would result in out-of-resonance forcing. The LHS of (44) can then be approximated by a white noise of identical spectral density at angular frequency $n \omega$, i.e.,

$$
\begin{aligned}
& \frac{\mathrm{d}^{2} \bar{\eta}_{n}}{\mathrm{~d} t^{2}}+\sigma \frac{\mathrm{d} \bar{\eta}_{n}}{\mathrm{~d} t}+(n \omega)^{2} \bar{\eta}_{n}=\zeta_{n}(t) \\
& \left\langle\zeta_{n}(t) \zeta_{n}(t+\tau)\right\rangle=\frac{C(n)}{2}\left(\frac{2 n n_{\mathrm{f}} \omega^{2}}{L \mathcal{D}}\right)^{2} \delta(\tau) \\
& C(n)=S_{\mathrm{w}}\left(\left|n-n_{\mathrm{f}}\right| \omega\right)+S_{\mathrm{w}}\left(\left(n+n_{\mathrm{f}}\right) \omega\right)
\end{aligned}
$$

We obtain a Langevin equation where the dissipation and injection processes are of distinct origin and are not linked 
by a fluctuation-dissipation relation. This system can be fully characterized, in particular the mean energy at a scale $n$ is found to be

$$
\left\langle E_{n}\right\rangle=\frac{L(n \omega)^{2} \eta_{\mathrm{f}}^{2}\left\langle\bar{\eta}_{n}^{2}\right\rangle}{2}=\frac{L \pi}{4 \sigma} \frac{C(n)}{2}\left(\frac{2 n n_{\mathrm{f}} \omega^{2} \eta_{\mathrm{f}}}{L \mathcal{D}}\right)^{2} .
$$

The statistics of the injected power fluctuation by radiative pressure can also be computed, see [18].

As an example, we detail the case of a low-frequency forcing $\left(n_{\mathrm{f}}=1\right)$ and a low-frequency motion of the boundary,

$$
S_{\mathrm{w}}(\tilde{w})=\frac{2 \sqrt{2}\left\langle\xi(t)^{2}\right\rangle}{\pi \omega\left(\left(1-\left(\frac{\tilde{\omega}}{\omega}\right)^{2}\right)^{2}+2\left(\frac{\tilde{\omega}}{\omega}\right)^{2}\right)} .
$$

Equation (49) stands for a second-order low-pass filter with a cut-off frequency equal to $\omega$ and a quality factor $1 / \sqrt{2}$. This is a possible thermal equilibrium spectrum of the wall as long as it is not perturbed by the radiative pressure. $\left\langle\xi(t)^{2}\right\rangle$ is then proportional to the temperature of the wall. According to (48), the energy spectrum of the waves at large frequencies compared to the cut-off one $(n \gg 1)$ is

$$
\left\langle E_{n}\right\rangle=\frac{2 \sqrt{2}\left\langle\xi(t)^{2}\right\rangle \omega^{3} \eta_{\mathrm{f}}^{2}}{n^{2} \mathcal{D}^{3} c} .
$$

In particular, $\left\langle E_{n}\right\rangle \propto \eta_{\mathrm{f}}^{2}$ results from the linearity of the initial equations and $\left\langle E_{n}\right\rangle \propto n^{-2}$ is a consequence of the second-order nature of the noise $S_{\mathrm{w}}$. The proportionality $\left\langle E_{n}\right\rangle \propto\left\langle\xi(t)^{2}\right\rangle$ differs from the periodic motion and is a sign of the loss of temporal coherence of the forcing. Finally, $\left\langle E_{n}\right\rangle \propto \mathcal{D}^{-3}$ shows that this effect will be of importance in high Q-factor cavities.

The validity of (44) can be checked afterwards by comparing the spectral power density of the considered forcing term with the neglected one. We get

$$
\frac{S_{\mathrm{W}}(n \omega) \omega^{2}\left\langle\eta_{+}(L, t)^{2}\right\rangle}{S_{\mathrm{w}}(n)(n \omega)^{2}\left\langle\bar{\eta}_{n}(L, t)^{2}\right\rangle} \sim \frac{\mathcal{D}}{\left(\frac{n \omega}{c}\right)^{2}\left\langle\xi(t)^{2}\right\rangle},
$$

and eq. (50) is then valid in the limit of small vibration of the boundary.

The stochastic and periodic regimes have therefore specific features, including the absence or presence of shock waves and the scaling of the energy spectra. This traces back to the temporal coherence of the forcing and to the nature of the energy transfers: the $n$-th mode is directly coupled with the forcing scale in the noisy case, whereas it is driven by the $(n \pm 1)$-th mode if the motion is harmonic.

Conclusion. - We have described how the oscillation of a boundary affects the reflection of surface waves. We experimentally evidenced this generalized Doppler effect, then computed it for gravity waves and discussed a model in which the cumulative effects of such interactions can be studied in a simple manner. Since self-similar spectra have been found to arise as a consequence of the Doppler effect, the comparison of such energy transfers with the non-linear ones would be an interesting follow-up in order to clarify experiments of surface wave turbulence. We finally detail possible applications of our study in two domains other than hydrodynamics.

First, in electrodynamics, where the motion of mirrors has been extensively discussed when it spontaneously generates photons from vacuum fluctuations. For this effect to be sizable, the mirror has to move at a velocity close to the speed of light, hence the resort to other devices in practice (e.g., superconducting circuits, see [19]). Since the aim of our last model was not to investigate the quantum creation of photons but to describe how energy can be generated at frequencies different from the forcing one, an additional injection mechanism acting as a source of photons was set. This process no longer requires high velocities to be relevant, especially since the development of high Q-factor cavities (a striking example being the ones used to test quantum mechanics principles [20], where photons of frequency $f \sim 50 \mathrm{GHz}$ are trapped in cavities with $Q$ up to $\left.3.10^{8}\right)$. The present study can be used, for instance, to know how the electromagnetic field is affected by the thermal motion of a Fabry-Pérot interferometer with a high coefficient of finesse.

Secondly, in acoustics, where a system very similar to the model presented here is commonly used in laboratories under the name of acousto-optic modulator, or "Bragg cell". Proposed by Brillouin nearly a century ago [21], it consists of a cavity where acoustic waves are generated by a periodically oscillating surface (a piezoelectric transducer) and are reflected at the other extremity by a fixed wall. Contrary to the idealisation of linear acoustics presented here, bulk non-linearities may have to be taken into account and have been studied using devices in which reflection at one extremity is cancelled [22]. In the usual Bragg cell configuration, our model shows that a forcing at a resonant frequency leads to the apparition of strong pressure gradients and our results can be used to predict whether or not non-linearities have to be considered.

The author is thankful to S. FAUVE, F. PÉTRÉLIS and B. GALLET for fruitful discussions. This work is supported by CNES and ANR-12-BS04-0005-02.

\section{REFERENCES}

[1] Censor D., J. Franklin Inst., 295 (1973) 103.

[2] Censor D., J. Acoust. Soc. Am., 83 (1988) 1223.

[3] Mujica N., Wunenburger R. and Fauve S., Eur. Phys. J. B, 33 (2003) 209.

[4] Falcon E., Laroche C. and Fauve S., Phys. Rev. Lett., 98 (2007) 094503.

[5] Nazarenko S., Wave Turbulence (Springer, Heidelberg) 2011.

[6] Dittrich J., Duclos P. and Šeba P., Phys. Rev. E, 49 (1994) 3535.

[7] Law C. K., Phys. Rev. Lett., 73 (1994) 1931.

[8] Méplan O. and Gignoux C., Phys. Rev. Lett., 76 (1996) 408.

[9] Dodonov V. V., Phys. Scr., 82 (2010) 038105. 
[10] Michel G., Pétrélis F. and Fauve S., Phys. Rev. Lett., 116 (2016) 174301.

[11] Censor D., J. Acoust. Soc. Am., 76 (1984) 1527.

[12] Havelock T. H., Philos. Mag., 8 (1929) 569.

[13] Joo S. W., Schyltz W. W. and Messiter A. F., J. Fluid Mech., 214 (1990) 161.

[14] Ting C-L and Perlin M., J. Fluid Mech., 295 (1995) 263.

[15] Rhodes-Robinson P. F., Math. Proc. Cambridge Philos. Soc., 92 (1982) 369.

[16] Biferale L., Annu. Rev. Fluid Mech., 35 (2002) 441.

[17] Dallas V., Fauve S. and Alexakis A., Phys. Rev. Lett., 115 (2015) 204501.

[18] Farago J., J. Stat. Phys., 107 (2002) 781.

[19] Wilson C. M. et. al., Nature, 479 (2011) 367.

[20] Raimond J. M., Brune M. and Haroche S., Rev. Mod. Phys., 79 (2001) 565.

[21] Brillouin L., Ann. Phys., 17 (1922) 88.

[22] Adler L. and Hiedermann E. A., J. Acoust. Soc. Am., 34 (1962) 410. 